OPEN ACCESS

Edited by:

Ulrike Kappler,

The University of Queensland,

Australia

Reviewed by:

Lars Ingo Ole Leichert,

Ruhr University Bochum, Germany

Wolfgang Buckel,

University of Marburg, Germany

${ }^{*}$ Correspondence:

Dan Su

sudan@scu.edu.cn

Specialty section:

This article was submitted to Microbial Physiology and Metabolism,

a section of the journal

Frontiers in Microbiology

Received: 14 May 2019

Accepted: 22 August 2019 Published: 06 September 2019

Citation:

Chen $Y$, Chen $Y$, Jiang $H, L u D$, Hu T, Bi G, Ran Y, Yu B, Dong H and Su D (2019) A Unique

Homo-Hexameric Structure of 2-Aminomuconate Deaminase in the Bacterium Pseudomonas

species AP-3.

Front. Microbiol. 10:2079. doi: 10.3389/fmicb.2019.02079

\section{A Unique Homo-Hexameric Structure of 2-Aminomuconate Deaminase in the Bacterium Pseudomonas species AP-3}

\author{
Yanjuan Chen ${ }^{1}$, Yiping Chen ${ }^{1}$, Hua Jiang ${ }^{1}$, Deren $\mathrm{Lu}^{1}$, Tingting $\mathrm{Hu}^{1}$, Gang Bi ${ }^{1}$, \\ Yuping Ran', Baofeng Yư ${ }^{3}$, Hui Dong ${ }^{4}$ and Dan Su${ }^{1 *}$
}

${ }^{1}$ State Key Laboratory of Biotherapy and Cancer Center, West China Hospital, Sichuan University and Collaborative Innovation Center of Biotherapy, Chengdu, China, ${ }^{2}$ Department of Dermatovenereology, West China Hospital, Sichuan University, Chengdu, China, ${ }^{3}$ Department of Biochemistry and Molecular Biology, Basic Medical College, Shanxi Medical University, Taiyuan, China, ${ }^{4}$ Key Laboratory of Tianjin Radiation and Molecular Nuclear Medicine, Institute of Radiation Medicine, Chinese Academy of Medical Sciences and Peking Union Medical College, Tianjin, China

The bacterium Pseudomonas species sp. AP-3 is one of several microorganisms that are capable of using 2-aminophenol as its sole source of carbon, nitrogen and energy. Several 2-aminophenol-metabolizing enzymes have pivotal roles in the biodegradation of aniline and its derivatives as environmental pollutants in Pseudomonas. The bacterium Pseudomonas sp. AP-3 recruits a unique 2-aminomuconate deaminase (AmnE) to hydrolyze 2-aminomuconate to 4-oxalocrotonate, and releases ammonia in the modified meta-cleavage pathway by forming various compounds-including acetaldehyde, pyruvic acid, acetyl-CoA, and succinate-that may enter the Krebs cycle. AmnE also belongs to the YjgF/YER057c/UK114 family (also known as the Rid family), which is conserved in all domains of life and prefers structurally homotrimeric forms with diverse functional purposes. To study the mechanism of the modified meta-cleavage pathway in Pseudomonas sp. AP-3, we determined the first crystal structure of AmnE from Pseudomonas sp. AP-3 at $1.75 \AA$. AmnE forms a unique homohexamer instead of a trimer which is normally adopted by the members of YjgF/YER057c/UK114 family. Based on the structure of the AmnE hexamer, we observed a hydrophobic base composed of six Lp3 loops (residues 122-131) in each of the AmnE protomers that have pivotal roles in the assembly of the hexamer. Eighteen hydrogen bonds formed by the residues $\mathrm{Met}^{96}$, $\mathrm{Pro}^{126}$, and $\mathrm{Arg}^{56}$, which surround the hydrophobic base, allowed the combination of the two trimers into a stable hexamer. The single mutant of AmnE R56A lost the ability to maintain the hexameric conformation, and revealed that the hydrogen bonds between residues $\mathrm{Arg}^{56}$ and $\mathrm{Met}^{96}$ have pivotal roles in the AmnE hexameric assembly.

Keywords: homo-hexamer, 2-aminomuconate deaminase, YjgF/YER057c/UK114 family, RidA subfamily, Pseudomonas species AP-3

Abbreviations: AmnE, 2-aminomuconate deaminase; SEC, size-exclusion chromatography. 


\section{INTRODUCTION}

Many bacteria, including numerous species of Pseudomonas, possess genes for the degradation of aromatic compounds through the meta-cleavage pathway. Pseudomonas species AP-3 (Pseudomonas sp. AP-3) was isolated by growth based on the use of 2-aminophenol as the sole carbon, nitrogen, and energy source (Takenaka et al., 1997). A modified meta-cleavage pathway for 2-aminophenol metabolism has been studied comprehensively. This pathway is similar to that known for the meta-cleavage of catechol, except that one of the hydroxyl groups on the metabolites is replaced by an amino group (Takenaka et al., 1998). The 2-aminophenol is degraded by Pseudomonas sp. AP-3 via 2-aminomuconate-6-semialdehyde to 4-hydroxy-2-oxovalerate, which is further degraded to pyruvate and acetyl-CoA via the modified meta-cleavage pathway (Figure 1). A $13.9 \mathrm{~kb}$ region of the $A P-3$ strain encompasses eight tight clusters of genes designed in the form of $a m n[A-H]$. Each gene in the modified meta-cleavage pathway is similar to the corresponding gene that operated in the meta-cleavage pathway, except for the $a m n E$ gene. The $a m n E$ gene product AmnE is a deaminase, and hydrolyzes 2-aminomuconate to 4-oxalocrotonate and releases ammonia in the modified meta-cleavage pathway (Takenaka et al., 2000). With the inclusion of the AmnE, the metabolic pathways of 2-aminophenol and its derivatives in Pseudomonas sp. AP-3 consist of seven major enzymes translated from the amn gene cluster $(a m n A-a m n H)$ into a single operon, including 2-aminophenol 1,6-dioxygenase (AmnBA), 2-aminomuconic 6-semialdehyde dehydrogenase (AmnC), 4-oxalocrotonate decarboxylase (AmnD), 2-aminomuconate deaminase (AmnE), 2-oxopent-4-enoate hydratase (AmnF), 4-hydroxy-2-oxovalerate aldolase $(\mathrm{AmnG})$, and acetaldehyde dehydrogenase $(\mathrm{AmnH})$. Zhongqi $\mathrm{H}$. and Jim C. S. also revealed the mechanism of the deamination reaction in the modified meta-pathway and identified the 2-aminomuconate deaminase acted specifically on the unsaturated $\alpha$-amino acid 2-aminomuconate in P. pseudoalcaligenes JS45 (He and Spain, 1998). The deduced amino acid sequence of 2-aminomuconate deaminase in Pseudomonas sp. AP-3 shows that AmnE belongs to a member of the YjgF/YER057c/UK114 protein family.

The YjgF/YER057c/UK114 protein family (also known as the Rid family) includes 200 prokaryotic and eukaryotic proteins, and consists of 130 amino acid residues $(\sim 15 \mathrm{kDa})$ which are found in all three domains of life (Kim et al., 2001). Phylogenetic analysis divided this family into a widely distributed archetypal RidA subfamily, and into seven other subfamilies (Rid1-Rid7). The archetypal RidA subfamily is found in all domains, while the Rid1-7 subfamilies are present only in prokaryotes. These proteins have widespread roles in metabolic processes in nearly every organism, and some species encode multiple members (Niehaus et al., 2015). In bacteria and yeast, protein family members exhibit predefined roles in the biosynthesis of essential compounds. Conversely, the mammalian members involved in the regulation of cellular differentiation inhibit translations by degrading messenger ribonucleic acid (Schmiedeknecht et al., 1996). After decades of research, 34 structures, including AmnE in the RidA subfamily, have been

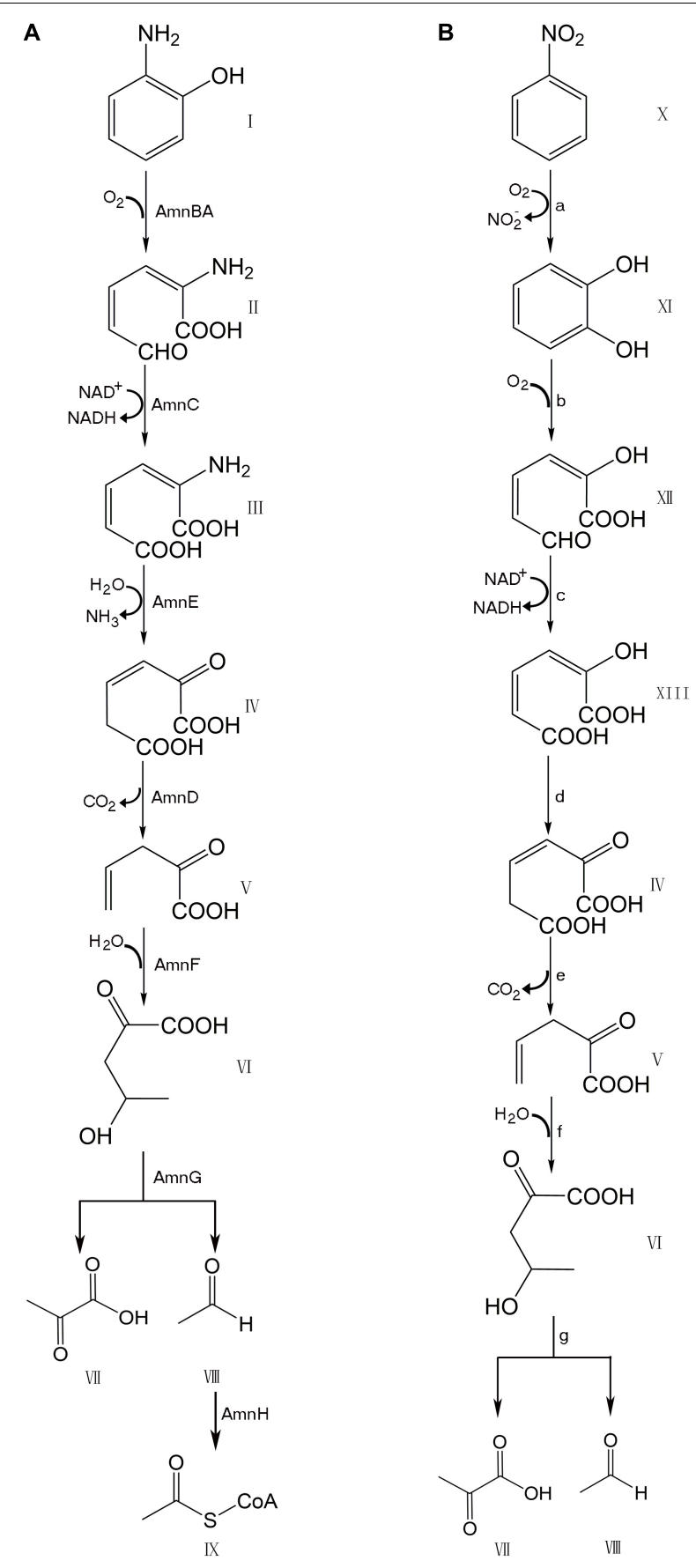

FIGURE 1 | Comparison of modified meta-cleavage pathway in Pseudomonas species AP-3 and meta-cleavage pathway in Comamonas species JS765. (A) Modified meta-cleavage pathway in Pseudomonas species AP-3. AmnBA: 2-aminophenol 1,6-dioxygenase. AmnC:

2-aminomuconic 6-semialdehyde dehydrogenase. AmnE: 2-aminomuconate deaminase. AmnD: 4-oxalocrotonate decarboxylase. AmnF:

2-oxopent-4-enoate hydratase. AmnG: 4-hydroxy-2-oxovalerate aldolase. $\mathrm{AmnH}$ : acetaldehyde dehydrogenase. (B) Meta-cleavage pathway in Comamonas species JS765. a: nitrobenzene dioxygenase. b: catechol 2,3-dioxygenase. c:2-hydroxymuconic semialdehyde dehydrogenase. d: 4-oxalocrotonate tautomerase. e:4-oxalocrotonate decarboxylase. f: 2-oxo-4-pentenoate hydratase. g:4-hydroxy-2-oxovalerate aldolase. 


\section{FIGURE 1 | Continued}

I: 2-aminophenol. II: 2-aminomuconic 6-semialdehyde. III: 2-aminomuconic acid. IV: 2-oxo-3-hexene-1,6-dioate. V: 2-oxopent-4-enoic acid. VI: 4-hydroxy-2-oxovalerate. VII: Pyruvic acid. VIII: Acetaldehyde. IX: Acetyl-CoA. X: nitrobenzene. XI: catechol. XII: 2-hydroxymuconic semialdehyde. XIII: 2-hydroxymuconate. The figure was produced with ChemDraw Professional 15.1

identified and deposited in the protein data bank (PDB). Many other existing homologs have not been published yet. 31 structures in the RidA subfamily exhibit the preferential adoption of homotrimers and form a distinct inter-subunit cavity that may serve as a small molecular binding site, but with unknown functions. Based on the experimental evidence, the RidA subfamily members have been recently annotated as imine deaminase (Hodge-Hanson and Downs, 2017).

Although the 2-aminophenol metabolic pathway and the corresponding catabolic enzymes have been studied extensively, the knowledge of the structural basis of the catabolic enzymes is still lacking. In this study, we demonstrate that AmnE selfassembles in a unique homo-hexameric form that is different from a regular trimer model of the RidA subfamily. The homohexameric structure of AmnE has been determined at a high resolution (equal to $1.75 \AA$ ), and constitutes the first protein structure in the metabolic pathway of 2-aminophenol. We analyzed the structure of the AmnE hexamer and explained how the six identical protomers assembled into a stable homohexameric conformation. Meanwhile, we identified that the residues $\mathrm{Ala}^{49}-\mathrm{Pro}^{59}$ on the Lp2 loop region (residues 38-60) of AmnE have pivotal roles in the assembly of the hexameric structure. The single mutant of AmnE R56A lost the ability to maintain the hexameric conformation, and thus revealed that the hydrogen bonds between residues $\mathrm{Arg}^{56}$ and $\mathrm{Met}^{96}$ have pivotal roles in the AmnE hexameric assembly.

\section{MATERIALS AND METHODS}

\section{Generation of Wild Type and Correlated Mutant Plasmid Constructs}

The gene encoding deaminase AmnE from Pseudomonas sp. AP3 was synthesized by Sangon Biotech Co., Ltd., China, and was cloned into a pET-28a (GE Healthcare, United States) using a fast cloning method (Li et al., 2011). The single mutants AmnE R56A and AmnE P126A, and the truncated mutant AmnE $\Delta 49$ 59 were generated by designing primers that encoded the mutated amino acids. Mutagenetic constructs were confirmed by commercial deoxyribonucleic acid (DNA) sequencing (Sangon Biotech, China). The primers used in this study are listed in Table 1.

\section{Protein Expression and Purification}

Wild plasmids of the type AmnE and correlated mutants were transformed into the BL21 (DE3) pLysS strain (TransGen Biotech, China) and grown in Luria-Bertani (LB) medium containing kanamycin $(50 \mu \mathrm{g} / \mathrm{ml})$ and chloromycetin $(34 \mu \mathrm{g} / \mathrm{ml})$. Growing cells were induced at an optical density $\left(\mathrm{OD}_{600}\right)$ value in
TABLE 1 | Primers used in this study.

\begin{tabular}{|c|c|c|}
\hline Gene & Primer & Sequence $\left(5^{\prime}-3^{\prime}\right)$ \\
\hline AmnE & $\mathrm{HF}$ & $\begin{array}{l}\text { CTGGTGCCGCGCGGCAGCATGGTTAGCAAA } \\
\text { GCTGATAA }\end{array}$ \\
\hline AmnE & $\mathrm{HR}$ & $\begin{array}{l}\text { GATGATGATTCCTCCTCCTTAGAGCGGCT } \\
\text { TGTATGCCA }\end{array}$ \\
\hline AmnE $\Delta 49-59$ & $\mathrm{~F}$ & AATACGTTTGTGGGCAATATTGAGC \\
\hline AmnE $\Delta 49-59$ & $\mathrm{R}$ & GCCCACAAACGTATTATCGGGCCTG \\
\hline AmnE R56A & $\mathrm{F}$ & CCGGATGATACCGGTGCACCCAGGCCA \\
\hline AmnE R56A & $\mathrm{R}$ & CGACCGGTATCATCCGGTTCAGCGCCC \\
\hline AmnE P126A & $\mathrm{F}$ & GCCGTCCATCAACTGGCGCACCCTCAAT \\
\hline AmnE P126A & $\mathrm{R}$ & CGCCAGTTGATGGACGGCGACCGTGGTA \\
\hline
\end{tabular}

the range of $0.6-0.8$ by the addition of $0.5 \mathrm{mM}$ isopropyl- $\beta-\mathrm{D}-1$ thiogalactopyranoside (IPTG) for $16 \mathrm{~h}$ at $16^{\circ} \mathrm{C}$. Cells harvested by centrifugation for $10 \mathrm{~min}$ at 4,000 revolutions per minute (rpm) were suspended in lysis buffer (20 mM Tris, $200 \mathrm{mM}$ $\mathrm{NaCl}$, and $10 \mathrm{mM}$ imidazole, $\mathrm{pH}$ 8.0) and lysed using a highpressure homogenizer (JNBIO, China) at $4^{\circ} \mathrm{C}$. The cell lysate was centrifuged for $30 \mathrm{~min}$ at $15,000 \mathrm{rpm}$ at $4^{\circ} \mathrm{C}$ (Thermo Sorvall LYNX 6000, United States), and the supernatant was loaded onto a Ni-NTA affinity chromatography column (GE Healthcare, United States) which was equilibrated with a lysis buffer. The unbound proteins were washed away with wash buffer (20 mM Tris, $200 \mathrm{mM} \mathrm{NaCl}, 50 \mathrm{mM}$ imidazole, $\mathrm{pH}$ 8.0). The tightly bound proteins, which mainly comprised AmnE, were eluted with elution buffer $(20 \mathrm{mM}$ Tris, $200 \mathrm{mM} \mathrm{NaCl}$, and $250 \mathrm{mM}$ imidazole $\mathrm{pH}$ 8.0). The N-terminal $6 \times$ His tag was cleaved with Thrombin (Sigma, United States) in the dialysis buffer $(20 \mathrm{mM}$ Tris, $20 \mathrm{mM} \mathrm{NaCl}$, and $5 \%(\mathrm{v} / \mathrm{v})$ glycerol, $\mathrm{pH}$ 8.0) at $6^{\circ} \mathrm{C}$ overnight. The AmnE protein was further purified by anion-exchange chromatography on a Resource Q column (GE Healthcare, United States). The protein was then purified by size-exclusion chromatography using a Superdex75 10/300 GL column (GE Healthcare, United States). The single mutants AmnE R56A and AmnE P126A, and the truncated mutant AmnE $\triangle 49-59$ were pre-equilibrated with buffer (50 mM HEPES, $150 \mathrm{mM} \mathrm{NaCl}, 1 \mathrm{mM}$ DTT, $1 \mathrm{mM}$ EDTA, and 5\% (v/v) glycerol, $\mathrm{pH}$ 8.0). All proteins were concentrated by ultrafiltration using a Millipore centrifugal ultrafiltration device (Amicon Ultra, $3 \mathrm{kDa}$ cutoff, Germany) and analyzed using sodium dodecyl sulfate polyacrylamide electrophoresis (SDS-PAGE) (Supplementary Figures S1-S4). For crystallization purposes, the protein AmnE was concentrated to $10 \mathrm{mg} / \mathrm{ml}$.

\section{Crystallization, X-Ray Data Collection, and Structure Determination}

A Gryphon robot (Art Robbins Instruments, United States) was used for the initial screening of 96 well plates at $20^{\circ} \mathrm{C}$ with the sitting-drop vapor diffusion method. Drops were prepared by mixing $0.5 \mu \mathrm{l}$ of purified protein and $0.5 \mu \mathrm{l}$ of reservoir solution. Commercial crystallization kits (Crystal Screen, Crystal Screen 2, Index Screen, Salt screen, and PEG Screen) were bought from Hampton Research (United States). Protein crystals were obtained only at $0.2 \mathrm{M} \mathrm{MgCl}_{2} \bullet 6 \mathrm{H}_{2} \mathrm{O}, 0.1 \mathrm{M}$ Tris- $\mathrm{HCl}$ $\mathrm{pH}$ 8.5, 30\% (w/v) PEG 4000 (Supplementary Figure S5A). 
Optimization was carried out in 24-well plates by using hanging-drop method. Drops were mixed with $1 \mu \mathrm{l}$ of protein solution at $10 \mathrm{mg} / \mathrm{ml}$ and $1 \mu \mathrm{l}$ of reservoir solution. The high-quality crystals were obtained in $0.1 \mathrm{M}$ CAPSO [3(Cyclohexylamino)-2-hydroxy-1-propanesulfonic acid] $\mathrm{pH}$ 9.6, $0.2 \mathrm{M} \mathrm{MgCl}_{2} \bullet 6 \mathrm{H}_{2} \mathrm{O}$, and 30\% (w/v) PEG 4000 (Supplementary Figure S5B). The crystals were flash-cooled in liquid nitrogen and the diffraction data were collected in the Shanghai Synchrotron Radiation Facility (SSRF) Beamline BL19U1 (Shanghai, China). The data were processed with HKL2000 software (Otwinowski and Minor, 1997). The best crystal diffracted X-rays and formed a pattern with a high-resolution equal to $1.75 \AA$. The space group of AmnE is C121, with cell dimensions set at $a=170.871 \AA$, $b=54.781 \AA, c=134.594 \AA$ (Supplementary Figure S5C), while the AmnE structure was solved by molecular replacement method with the Phaser (Lebedev et al., 2008) in the CCP4 suite (Winn et al., 2011), using the initial model of Pyrococcus horikoshii-PH0854 (PDB: 2DYY). The refined model was further built manually according to the electron density map with COOT (Emsley and Cowtan, 2004), and the qualities of the refined models were assessed with PHENIX (Adams et al., 2010). The final structure was derived to a $\mathrm{R}_{\text {work }}$ factor of $15.7 \%\left(\mathrm{R}_{\text {free }}\right.$ factor of 19.5\%). All figures were generated by using PyMOL ${ }^{1}$. Data collection, statistics, and structure-refinement statistical outcomes are listed in Table 2.

\section{Dynamic Light Scattering}

Measurements were obtained using a Zetasizer $\mu \mathrm{V}$ (Malvern Panalytical, Worcestershire, United Kingdom) with AmnE at a concentration of $10 \mathrm{mg} / \mathrm{ml}$ in a buffer solution [50 mM HEPES, $150 \mathrm{mM} \mathrm{NaCl}, 1 \mathrm{mM}$ DTT, $1 \mathrm{mM}$ EDTA, and 5\% (v/v) glycerol, $\mathrm{pH}$ 8.0] at $4^{\circ} \mathrm{C}, 16^{\circ} \mathrm{C}, 20^{\circ} \mathrm{C}$, and $25^{\circ} \mathrm{C}$, separately. The results were analyzed using software provided by the manufacturer. Experimental errors were estimated based on the estimated standard deviations.

\section{Analytic Size-Exclusion Chromatography}

Analytic size-exclusion chromatography (SEC) was adopted to investigate the oligomeric state of AmnE and associated mutants using a Superdex 200 10/300 GL column (GE Healthcare, United States). The column was pre-equilibrated with an analysis buffer [50 mM HEPES, $150 \mathrm{mM} \mathrm{NaCl}, 1 \mathrm{mM}$ DTT, 1 mM EDTA, and $5 \%(\mathrm{v} / \mathrm{v})$ glycerol, $\mathrm{pH} 8.0 \mathrm{]}$. Furthermore, all the samples, at a concentration of $1 \mathrm{mg} / \mathrm{ml}$, were analyzed at a flow rate of $0.3 \mathrm{ml} / \mathrm{min}$ at $16^{\circ} \mathrm{C}$. The molecular mass was calculated using the following equation,

$$
V e=-b^{\prime} \lg M r+c^{\prime}
$$

$V e$ : the volume at which the intermediate molecules elute; Mr: molecular mass.

\section{Chemical Cross-Linking Assay}

AmnE wild type and mutant proteins were purified with the use of a Superdex 75 10/300 GL column (GE Healthcare,

${ }^{1}$ http://www.pymol.org
TABLE 2 | Data-collection and relevant statistics (values in parentheses correspond to the shell with the highest resolution).

\begin{tabular}{|c|c|}
\hline & AmnE \\
\hline \multicolumn{2}{|l|}{ Data collection } \\
\hline Wavelength $(\AA)$ & 0.9789 \\
\hline Beamline & BL19U1 \\
\hline Detector & CCD Pilatus CBF \\
\hline Space group & C121 \\
\hline Unit-cell parameters $\left(\AA{ }^{\circ}\right)$ & $\begin{array}{c}a=170.871, b=54.781 \\
c=134.594 ; \alpha=90.00 \\
\beta=101.89, \gamma=90.00\end{array}$ \\
\hline Resolution $(\AA)$ & $50.00-1.76(1.76-1.81)$ \\
\hline $\mathrm{R}_{\text {merge }}(\%)^{\dagger}$ & $90.1(53.5)$ \\
\hline Average $I / \sigma(l)$ & $28.25(1.94)$ \\
\hline No. of observed reflections & $234852(19456)$ \\
\hline No. of unique reflections & 120742 (9970) \\
\hline Completeness (\%) & $99.3(99.0)$ \\
\hline Redundancy & $3.4(3.3)$ \\
\hline Matthews coefficient $\left(\AA^{3} \mathrm{Da}^{-1}\right)$ & 2.32 \\
\hline Solvent content (\%) & 47 \\
\hline Molecules per asymmetric unit & 9 \\
\hline \multicolumn{2}{|l|}{ Refinement } \\
\hline Resolution $(\AA)$ & $49.511-1.754$ \\
\hline $\mathrm{R}_{\text {work }} / \mathrm{R}_{\text {free }}$ & $0.1570 / 0.1949$ \\
\hline Ramachandran favored (\%) & 96.56 \\
\hline Ramachandran allowed (\%) & 3.44 \\
\hline Ramachandran outliers (\%) & 0.00 \\
\hline \multicolumn{2}{|l|}{ No. of atoms } \\
\hline Protein & 9628 \\
\hline Ligand & 2 \\
\hline Water & 1042 \\
\hline Wilson B value & 22.69 \\
\hline \multicolumn{2}{|l|}{ Root-mean-square deviations } \\
\hline Bond lengths $(\AA)$ & 0.007 \\
\hline Bond angles $\left(^{\circ}\right)$ & 0.980 \\
\hline
\end{tabular}

${ }^{\dagger} R_{\text {merge }}=\sum_{h k l} \sum_{i}\left|\mathrm{l}_{i}(h k l)-\langle\mathrm{l}(h k l)\rangle\right| / \sum_{\mathrm{hkl}} \sum_{i} \mathrm{l}_{i}(h k l)$, where $\mathrm{l}_{i}(h k l)$ is an individual intensity measurement and $\langle\mathbf{l}(\mathrm{hkl})\rangle$ is the average intensity for all reflections.

United States). Glutaraldehyde (25\%) (Sigma, United States) was diluted at various concentrations $(0.1,0.2,0.3,0.4,0.5,0.6,0.7$, $0.8,0.9$, and $1 \%)$ in distilled water. Proteins in buffer $[50 \mathrm{mM}$ HEPES, $150 \mathrm{mM} \mathrm{NaCl}, 1 \mathrm{mM}$ DTT, $1 \mathrm{mM}$ EDTA, and 5\% (v/v) glycerol, $\mathrm{pH}$ 8.0] were incubated with glutaraldehyde at $25^{\circ} \mathrm{C}$ for $30 \mathrm{~min}$. The reaction was then quenched with the addition of $10 \mu \mathrm{l}$ solution of $1 \mathrm{M}$ Tris- $\mathrm{HCl}(\mathrm{pH} 8.0)$, and $5 \times$ SDS-PAGE loading buffer. All samples were analyzed on SDS-PAGE gels.

\section{Spectroscopy}

Before CD measurements, all proteins were purified using a Superdex 75 10/300 GL column (GE Healthcare, United States) in buffer [50 mM HEPES, $150 \mathrm{mM} \mathrm{NaCl}, 1 \mathrm{mM}$ DTT, $1 \mathrm{mM}$ EDTA, 5\% (v/v) glycerol, pH 8.0]. The CD spectra were recorded on a Jasco J-715 Spectropolarimeter (JASCO, MD, United States) with the use of three scans on average within the $190-260 \mathrm{~nm}$ wavelength range from solutions in $10 \mathrm{~mm}$ path length quartz cuvettes at $25^{\circ} \mathrm{C}$. Raw ellipticity data $\theta_{\text {obs }}$ (in $10^{4}$ 
millidegrees) were converted to mean residue ellipticity $(\theta)$ (in $10^{4}$ millidegrees) using the formula,

$$
(\theta)=\frac{(\theta \text { obs } \times M R W \times 100)}{(c \times l)}
$$

where $\theta$ is the ellipticity in $10^{4}$ millidegrees, $l$ is the path length of the cuvette in $\mathrm{cm}, M R W$ is the mean residue weight, and $c$ is the concentration in $\mathrm{mg} / \mathrm{ml}$. Data were analyzed with Microsoft Office Excel and Origin 8.

\section{RESULTS}

\section{Protomeric Structure of AmnE}

The AmnE protomer is a 142 residues polypeptide folded into a single domain. The overall structure of the AmnE protomer shows an $\alpha+\beta$ fold with a $\beta$-sheet $\left(\beta_{1}-\beta_{6}\right)$, two $\alpha$-helices $\left(\alpha_{1}, \alpha_{4}\right)$, and two $3_{10^{-}}$ helices $\left(\alpha_{2}, \alpha_{3}\right)$, in an arrangement of $\beta_{1}$ (residues 9 -10), $\beta_{2}$ (residues 25-28), $\beta_{3}$ (residues 31-37), $\alpha_{1}$ (residues 61 -78), $\alpha_{2}$ (residues 83-85), $\beta_{4}$ (residues 86-93), $\beta_{3}$ (residues 96-98), $\alpha_{4}$ (residues 99-106), $\beta_{5}$ (residues 116-121), and $\beta_{6}$ (residues 132-139) (Figure 2A). Six $\beta$-strands are aligned in a single sheet, and two long $\alpha$-helices $\left(\alpha_{1}, \alpha_{4}\right)$ are arranged in an almost parallel orientation on one side of the $\beta$-sheet. Additionally, two $3_{10}$ helices are observed in the structure of which $\alpha_{2}$ is positioned at the end of $\alpha_{1}$, followed by the $\beta$-strand $\beta_{4}$. Accordingly, $\alpha_{3}$ is located at the N-terminus of the $\alpha$-helix $\left(\alpha_{4}\right)$. In the $\beta$-sheet, $\beta_{4}$ and $\beta_{5}$ are parallel to each other, and all other strands are antiparallel. Three large loops (Lp1-3) extend from one side of the $\beta$-sheet consisting of an unfolded region. The Lp1 (residues 11-24) and Lp3 (residues 122-131) are located separately between the two $\beta$-strands, $\left(\beta_{1}, \beta_{2}\right)$ and $\left(\beta_{5}, \beta_{6}\right)$. Lp2 (residues 38-60) is the longest loop and connects $\beta_{3}$ and $\alpha_{1}$ (Figure 2A).

The structure of the AmnE protomer is highly conserved in the YjgF/YER057c/UK114 family. The DALI search from the PDB database retrieved 50 homologs with similar structural forms in the case of the AmnE protomer. Three protein structures are chosen to represent all prokaryotic and eukaryotic proteins in this protein family. They include the human trichloroacetic acid-soluble protein p14.5 (hp14.5, PDB code: 1ONI), the E. coli TdcF protein (PDB code: 2UYN), and YEO7 in Saccharomyces cerevisiae (PDB code: 1JD1). The protomers of the three proteins are highly conserved structurally (Supplementary Figures S6A-C). Pairwise superposition of the three protomers yield root-mean-square deviation (RMSD) values equal to $0.682 \AA$ (for $84 \mathrm{C} \alpha$ atoms, AmnE/TdcF), $0.702 \AA$ (for $85 \mathrm{C} \alpha$ atoms, AmnE/YE07), and $0.850 \AA$ (for $79 \mathrm{C} \alpha$ atoms, AmnE/hp14.5). The region that exhibits considerable structural protein diversity is located in the unfolding region. The Lp2 of AmnE is along the disorder loop, and extends from the main body compared to the other three structures. The sequence alignment data revealed that residues $\mathrm{Ala}^{49}-\mathrm{Pro}^{59}$ are in a unique region in $\mathrm{AmnE}$, which is deficient in Tdcf, YE07, and p14.5 (Supplementary Figure S6G).

\section{Structural Basis of AmnE Trimer}

AmnE adopts the classic homotrimer formed by three protomers like the other members of the YjgF/YER057c/UK114 family (Figure 2B). The core of the homotrimer is composed of three $\beta$-sheets. Each one of these is closed into an empty triangular barrel-type structure and is surrounded by six $\alpha$-helices on its outer part. The inner surface of the barrel forms a highly hydrophobic environment composed of $\beta 3, \beta 4$, and $\beta 6$ strands. Viewed from the top to the bottom of the trimer, one end of the barrel is sealed off by three phenylalanines $\left(\mathrm{Phe}^{31}\right)$, while the other is sealed by a hydrophobic loop Lp3 from each of the three protomers (Supplementary Figure S7). Residues Leu ${ }^{32}$, $\mathrm{Val}^{86}, \mathrm{Val}^{87}, \mathrm{Leu}^{93}, \mathrm{Ile}^{132}$, and Ile ${ }^{134}$ on the inner surface of the barrel are conserved in all of the members of the RidA subfamily (Supplementary Figure S8A). The hydrophobic residues are distributed on the inner surface of the barrel to maintain the stability of the homotrimer of AmnE. The single protomeraccessible surface buried in the trimer is $1994.1 \AA^{2}$, and the proportion of the buried area to a protomer' total surface area is $25.89 \%$. On the outer parts of the trimer, the interfaces of the adjacent subunits form three equivalent clefts, i.e., $\mathrm{AB}, \mathrm{BC}$, and CA (Figures 3A-C). These clefts are formed by $\alpha_{4}, \beta_{4}$, and $\beta_{5}$ in one subunit, and $\beta_{2}, \beta_{3}$, and $\beta_{6}$, loop Lp1, and $\alpha_{4}$ in the adjacent subunit. Lp1 covers the cleft and is associated with elevated temperature factors and increased RMSD values among the three protomers. Comparison of the protein-ligand complex structures of hp14.5 (PDB code: $1 \mathrm{ONI}$ ) and TdcF (PDB code: $2 \mathrm{UYN}$ ), shows that the Lp1 of AmnE is potentially involved in unknown ligand binding (Supplementary Figure S9). The residues $\mathrm{Ser}^{35}$, Gly ${ }^{36}$, $\mathrm{Glu}^{88}, \mathrm{Gln}^{103}, \mathrm{Arg}^{117}$, and Lys ${ }^{135}$ in these three clefts are highly conserved in the YjgF/YER057c/UK114 family (Figure 3D). The areas of the three clefts (AB, BC, and CA) in AmnE were respectively, calculated and were equal to $760.43,760.47$, and $741.33 \AA^{2}$, respectively. These areas could provide ideal contact surfaces for small molecules with unknown functions. The DALI search from the PDB library lists 26 homologies of the AmnE trimer in YjgF/YER057c/UK114 family (Table 3). The structural superimposition suggests that the trimer is conserved in YjgF/YER057c/UK114 family proteins. The RMSD value estimated based on 192-290 C $\alpha$ atoms in these structures varied in the range of $0.67-1.89 \AA$, with a sequence identity in the range of $23.00-40.51 \%$. The superposing structures revealed that three Lp2 loops from each AmnE protomer extended out far from the main body of the trimer, and formed a unique conformation compared to other homologs in the YjgF/YER057c/UK114 family (Supplementary Figures S6D-F). Accordingly, the shape of the AmnE trimer resembled a three-legged structure.

\section{The Structure of AmnE Hexamer}

AmnE exists as a hexamer in solution and not in a trimeric state like the other members of the YjgF/YER057c/UK114 family. We identified the hexameric form of AmnE with the use of sizeexclusion chromatography and cross-linking methods. AmnE was eluted at $13.36 \mathrm{ml}$ on a Superdex 200 size-exclusion column, and its calculated molecular mass was $103.5 \mathrm{kDa}$, which was close to that of the hexamer. This finding was also supported by crosslinking data. The crystal structure of AmnE was solved 
A
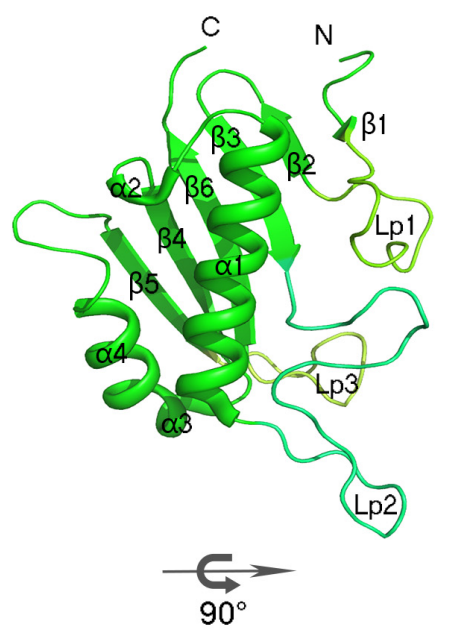

C

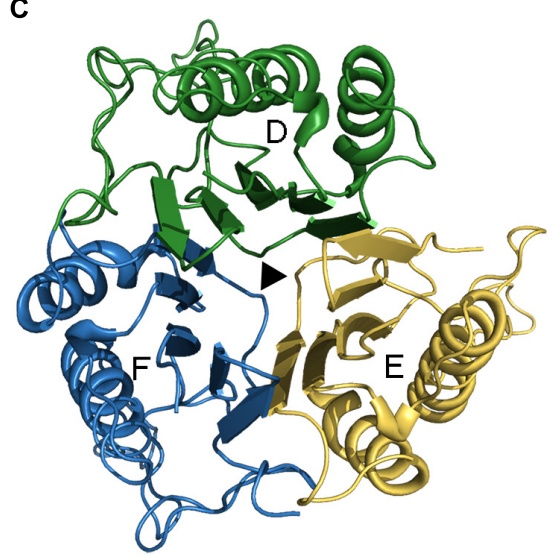

E

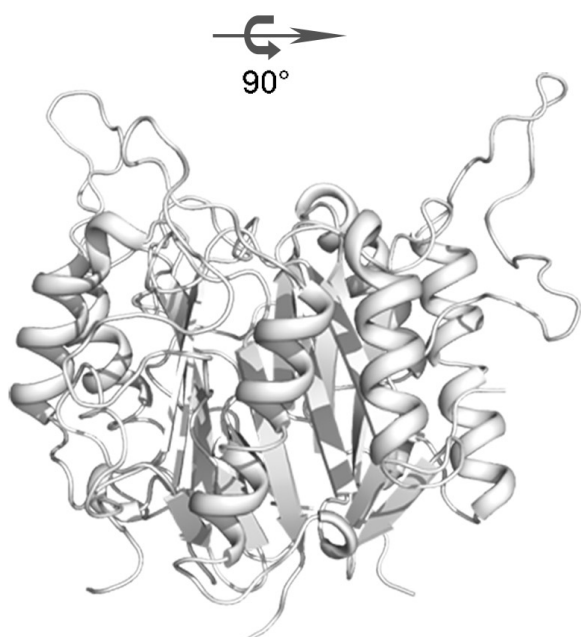

Trimer II

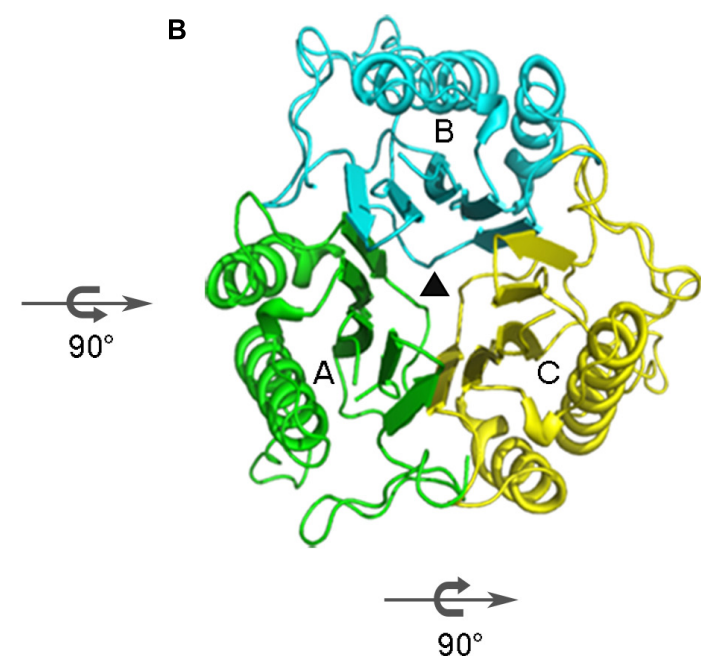

D
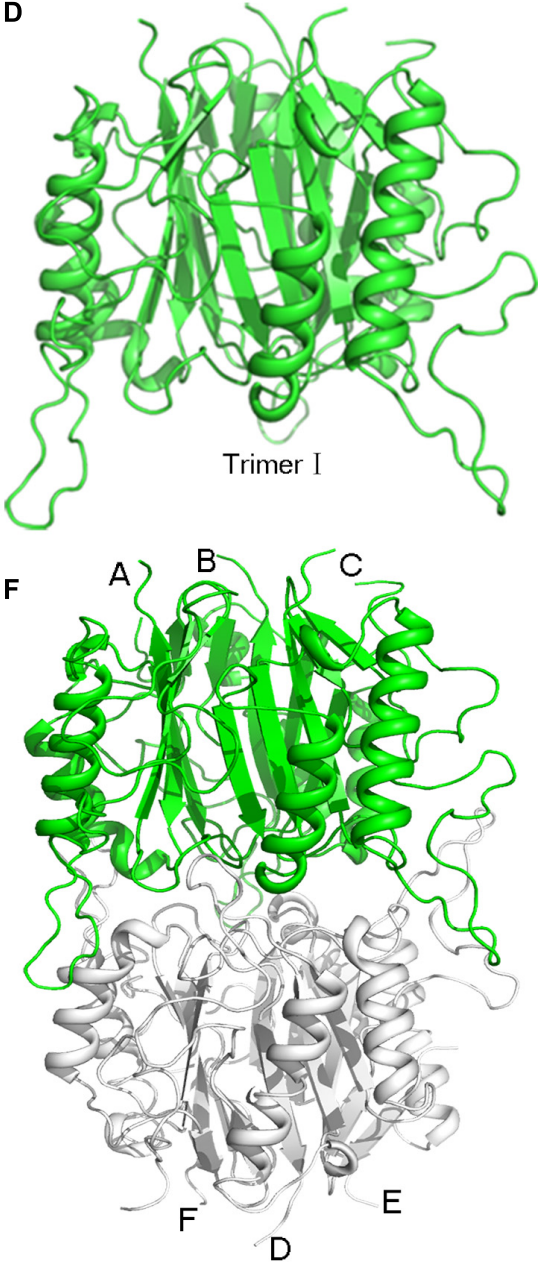

Hexamer

FIGURE 2 | Overview of the hexameric assembly of AmnE. (A) Three-dimensional view of the AmnE protomer. The ribbon diagram is colored in green. (B) AmnE trimer I (A/B/C). Subunits are drawn in different colors in this ribbon diagram viewed along the threefold axis. Chains $\mathrm{A}-\mathrm{C}$ are respectively, colored in green, cyan, and yellow. (C) AmnE timer II (D/E/F). Subunits are drawn in different colors in this ribbon diagram viewed along the threefold axis. Chains $D-F$ are respectively, colored in forest green, yellow orange, and sky-blue. (D-F) The AmnE hexamer is composed of two identical trimers (I and II). Trimer I is colored in green and trimer II is colored in gray. The figure was produced with PyMOL (http://www.pymol.org). 


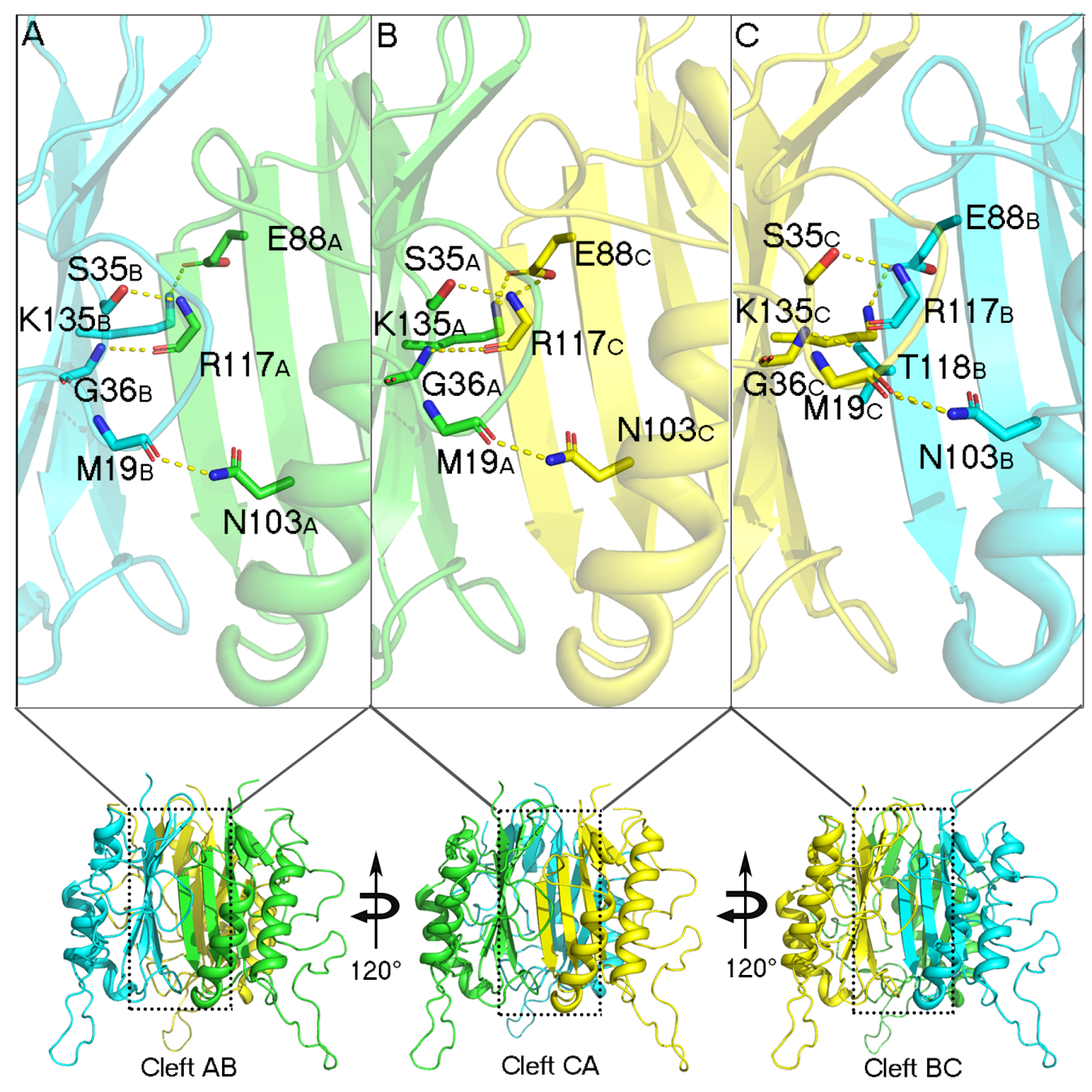

$\underset{A m n E}{D}$
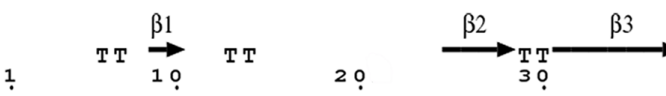

$40 \mathrm{~T}$

$T_{50} T_{T}$

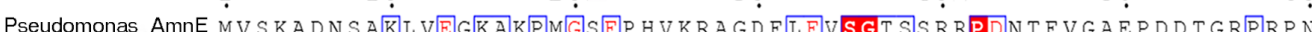

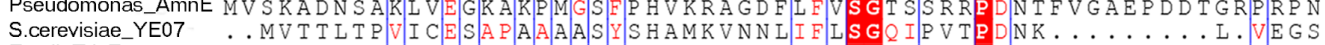

E.coli_TdCF

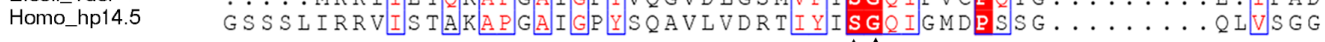

$\begin{array}{ccccc}\alpha 1 & \eta 1 & \beta 4 & \boldsymbol{\Delta}_{\eta 2} & \alpha 2\end{array}$

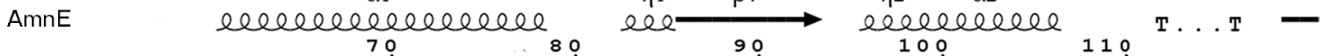

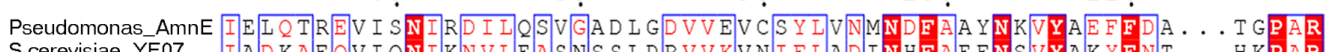

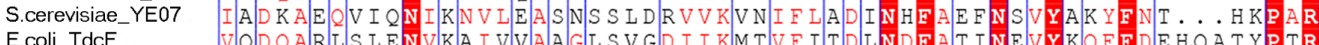

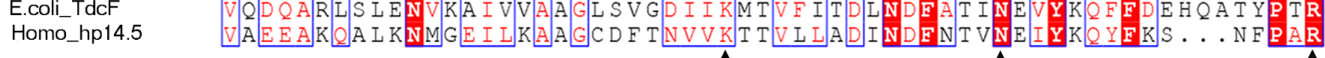

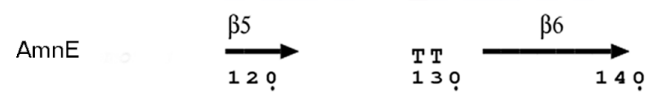

Pseudomonas_AmnE T T VAVH 0 LPH P Q L V I IT K VVAY K P L . . . .

S.cerevisiae_YE07 SCVIAVAALPLGVD.MEMEAIAAERD . . . .

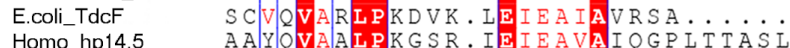

Homo_hp14.5 AAYQVAALPKGSR. IEIEAVAIQGPLTTASL

FIGURE 3 | Analyses of AmnE trimeric clefts. Conserved residues $\mathrm{Ser}^{35}$, Gly ${ }^{36}, \mathrm{Glu}^{88}, \mathrm{Gln}^{103}$, $\mathrm{Arg}^{117}$, and $\mathrm{Lys}^{135}$, are highlighted in the three clefts with sticks. (A) Interface of cleft AB. (B) Interface of cleft CA. (C) Interface of cleft BC. (D) Comparison of AmnE with the other three members of the YjgF/YER057c/UK114 family. Sequence alignment of AmnE from Pseudomonas sp. AP-3 with YE07 from Saccharomyces cerevisiae (34.00\% sequence identity), TdcF from Escherichia coli (29.00\% sequence identity), and hp14.5 from homo sapiens (28.72\% sequence identity). Residues labeled with triangles are conserved residues involved in the ligand binding pocket. Sequences were aligned using the tool obtained from the web site (https://www.ebi.ac.uk/Tools/msa/clustalo/) and the alignment was presented using the online ESPript 3.0 server (http://espript.ibcp.fr/ESPript/ESPript/). 
TABLE 3 | Homologous proteins of AmnE trimer.

\begin{tabular}{|c|c|c|c|c|}
\hline PDB code & Species & $\mathbf{C} \alpha$ atoms & RMSD & $\begin{array}{l}\text { Sequence } \\
\text { identities }\end{array}$ \\
\hline $6 I \mathrm{ZH}$ & Pseudomonas sp. AP-3 & 423 & 0.000 & $100 \%$ \\
\hline 5YU2 & Staphylococcus aureus & 271 & 0.672 & $28.33 \%$ \\
\hline 1JD1 & Saccharomyces cerevisiae & 279 & 0.691 & $34.00 \%$ \\
\hline 3І7Т & Mycobacterium tuberculosis & 260 & 0.721 & $32.00 \%$ \\
\hline 2B33 & Thermotoga maritima & 263 & 0.735 & $33.06 \%$ \\
\hline 2UYP & Escherichia coli & 252 & 0.760 & $29.00 \%$ \\
\hline $2 U Y K$ & Escherichia coli & 251 & 0.767 & $29.00 \%$ \\
\hline $3 L 7 Q$ & streptococcus mutans & 274 & 0.800 & $34.71 \%$ \\
\hline 1NQ3 & Capra hircus & 257 & 0.800 & $35.06 \%$ \\
\hline 1QAH & Rattus norvegicus & 270 & 0.807 & $28.00 \%$ \\
\hline 1QD9 & Bacillus subtilis (strain 168) & 252 & 0.830 & $40.51 \%$ \\
\hline $1 X R G$ & Clostridium thermocellum & 262 & 0.842 & $31.97 \%$ \\
\hline $5 Y 6 U$ & Bacillus subtilis (natto) & 254 & 0.849 & $40.51 \%$ \\
\hline 1QU9 & Escherichia coli & 238 & 0.855 & $29.93 \%$ \\
\hline 3MQW & Entamoeba histolytica & 252 & 0.893 & $34.18 \%$ \\
\hline $1 \mathrm{ONI}$ & Homo sapiens & 247 & 0.900 & $28.72 \%$ \\
\hline $3 \mathrm{~V} 4 \mathrm{D}$ & Escherichia coli & 290 & 0.970 & $28.00 \%$ \\
\hline 2DYY & Pyrococcus horikoshii & 267 & 0.970 & $39.23 \%$ \\
\hline ЗКОТ & Pseudomonas syringae & 260 & 0.991 & $25.00 \%$ \\
\hline 1PF5 & Escherichia coli (strain K12) & 276 & 1.066 & $32.00 \%$ \\
\hline $5 \mathrm{~V} 4 \mathrm{~F}$ & Yersinia pestis & 262 & 1.163 & $28.00 \%$ \\
\hline 2CWJ & Aeropyrum pernix & 271 & 1.173 & $30.00 \%$ \\
\hline 3LME & Rhodopseudomonas palustris & 245 & 1.290 & $23.00 \%$ \\
\hline $1 \mathrm{~J} 7 \mathrm{H}$ & Haemophilus influenzae & 245 & 1.332 & $29.00 \%$ \\
\hline 3K12 & Pseudomonas aeruginosa & 224 & 1.337 & $32.32 \%$ \\
\hline 3GTZ & Salmonella typhimurium & 243 & 1.397 & $32.00 \%$ \\
\hline 3I3F & Giardia lamblia & 192 & 1.889 & $32.00 \%$ \\
\hline
\end{tabular}

in the space group $\mathrm{C} 121$ with nine protomers $(\mathrm{A}, \mathrm{B}, \mathrm{C}, \mathrm{D}, \mathrm{E}$, F, G, H, I) composed of three identical homotrimers (trimers I, II, and III) in one asymmetric unit. One of the AmnE hexamers is organized based on the use of two identical trimers, trimer I $(\mathrm{A} / \mathrm{B} / \mathrm{C})$ and trimer II (D/E/F) (Figures 2B-F). Trimer III forms a hexamer with the use of trimer III in the neighboring asymmetric unit in the crystal lattice. The quaternary structure of AmnE was analyzed by PDBePISA. The total accessible surface of the two trimers buried in the hexamer is very large and equal to $3954.1 \AA^{2}$. Correspondingly, the proportion of the buried area to the total area of the two trimers is $11.59 \%$.

In the AmnE hexameric structure, two trimers (trimer I, timer II) interact in a back-to-back manner through the hydrophobic region composed of six $\mathrm{Lp} 3$ loops $\left(\mathrm{Lp} 3_{A}, \mathrm{Lp} 3_{B}\right.$, and $\mathrm{Lp} 3_{C}$, from trimer I, and $\mathrm{Lp}{ }_{D}, \mathrm{Lp}_{E}, \mathrm{Lp} 3_{F}$, from trimer II). These loops are used to seal off the bottom of the hydrophobic channel in all of the trimers. Six residues $\mathrm{His}^{123}$ (His ${ }^{123}{ }_{A-F}$ ), located on the loops Lp3, form a hydrophobic base with residues $\mathrm{Val}^{122}{ }_{A-F}$, Leu ${ }_{A-F}^{125}$, and $\mathrm{Leu}^{130}{ }_{A-F}$ present in the AmnE hexamer (Figures 4A,B,D). Outside of the hexamer, six loops $\left(\mathrm{Lp} 2_{A}, \mathrm{Lp} 2_{B}\right.$, and $\mathrm{Lp} 2_{C}$, from trimer $\mathrm{I}$, and $\mathrm{Lp} 2_{D}, \mathrm{Lp} 2_{E}$, and $\mathrm{Lp} 2_{F}$, from trimer II) surround the hexamer and extend into the cleft area of the opposite trimer to form a solid football-like shape. Based on the structural analysis, the Lp2 (residues 49-59) was identified to play a pivotal role in the maintenance of the stability of the AmnE hexamer. There are six pivotal residues
$\operatorname{Arg}^{56}\left(\operatorname{Arg}^{56}{ }_{A-F}\right)$ located on loops Lp2 (Lp2 $\left.A_{-F}\right)$ that formed eighteen hydrogen bonds in the hexamer with residues Pro ${ }^{126}$ (Pro ${ }^{126}{ }_{A-F}$ ) and $\mathrm{Met}^{96}\left(\mathrm{Met}^{96}{ }_{A-F}\right.$ ) (Figure 4C).

To further confirm that the functions of $\mathrm{Lp} 2$ and $\mathrm{Arg}^{56}$ contribute to the maintenance of the conformation of the AmnE hexamer in vitro, the mutant proteins AmnE $\Delta \mathrm{Lp} 2$ (residues $\mathrm{Ala}^{49}-\mathrm{Pro}^{59}$ ) and AmnE R56A were prepared and measured with CD spectroscopy before subsequent experiments (Figures $\mathbf{5 A}, \mathbf{B}$ ). The oligomerization states of the wild type and mutants of AmnE were evaluated by SEC. The calculated molecular mass of the wild-type AmnE and mutant AmnE P126A were respectively equal to 103 and $96.3 \mathrm{kDa}$, and were close to the theoretical molecular mass of the hexamer (Figures 5C,D). However,

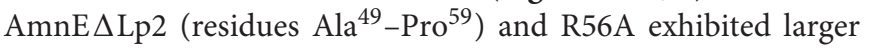
retention volumes, which corresponded to the molecular weight of the AmnE trimer (Figures 5E,F). Meanwhile, a glutaraldehyde crosslinking experiment was conducted to verify the multimeric state of each protein (Figures 5G-I). Primarily and foremost, we confirmed that the AmnE hexamer was in a stable state in solution, and that the residues $\mathrm{Arg}^{56}$ and $\mathrm{Met}^{96}$ located on the hexameric interface were two key residues required to maintain the hexameric conformation of AmnE.

\section{DISCUSSION}

The 2-aminophenol compounds are intermediates in the biodegradation of nitrobenzene. The benzene ring cleavage and deamination steps are essential in the mineralization of 2aminophenol for the growth of the assimilating bacteria. The metabolic pathway for 2-aminophenol in Pseudomonas sp. AP3 is referred to as the modified meta-cleavage pathway, in which 2 -aminomuconate is transformed into 4-oxalocrotonate by the deaminase AmnE (Takenaka et al., 2000). In this study, we elucidated the first structure of 2-aminomuconate deaminase involved in the 2-aminophenol pathway in Pseudomonas sp. AP3. AmnE also belongs to a member of the RidA subfamily in the YjgF/YER057c/UK114 family. In this family, proteins are distributed in all domains of life, apparently in the archetypal RidA subfamily and in seven other subfamilies. Members of the RidA subfamily prefer to form the trimeric, barrel-like quaternary structures, and inter-subunit cavities with diverse biological roles. Many of these are poorly understood despite their widespread existence (Sinha et al., 1999). In the PDB library, there are 33 structures of RidA subfamily in total, including one nuclear magnetic resonance (NMR) structure and 32 crystal structures. Most of these structures have not been published or characterized yet by the authors. Therefore, we expect to analyze all of the structures in the RidA subfamily and reveal that there are two primary types of homomultimeric protein complexes, i.e., trimeric and hexameric forms.

The trimeric conformation adopted by the RidA subfamily proteins is structurally conserved, and even the sequence similarity is comparatively low. These proteins adopt homotrimeric forms, and the clefts between the protomeric subunits are suggested to have functional relevance (Volz, 1999; Deaconescu et al., 2002; Deriu et al., 2003). The alignment of the 

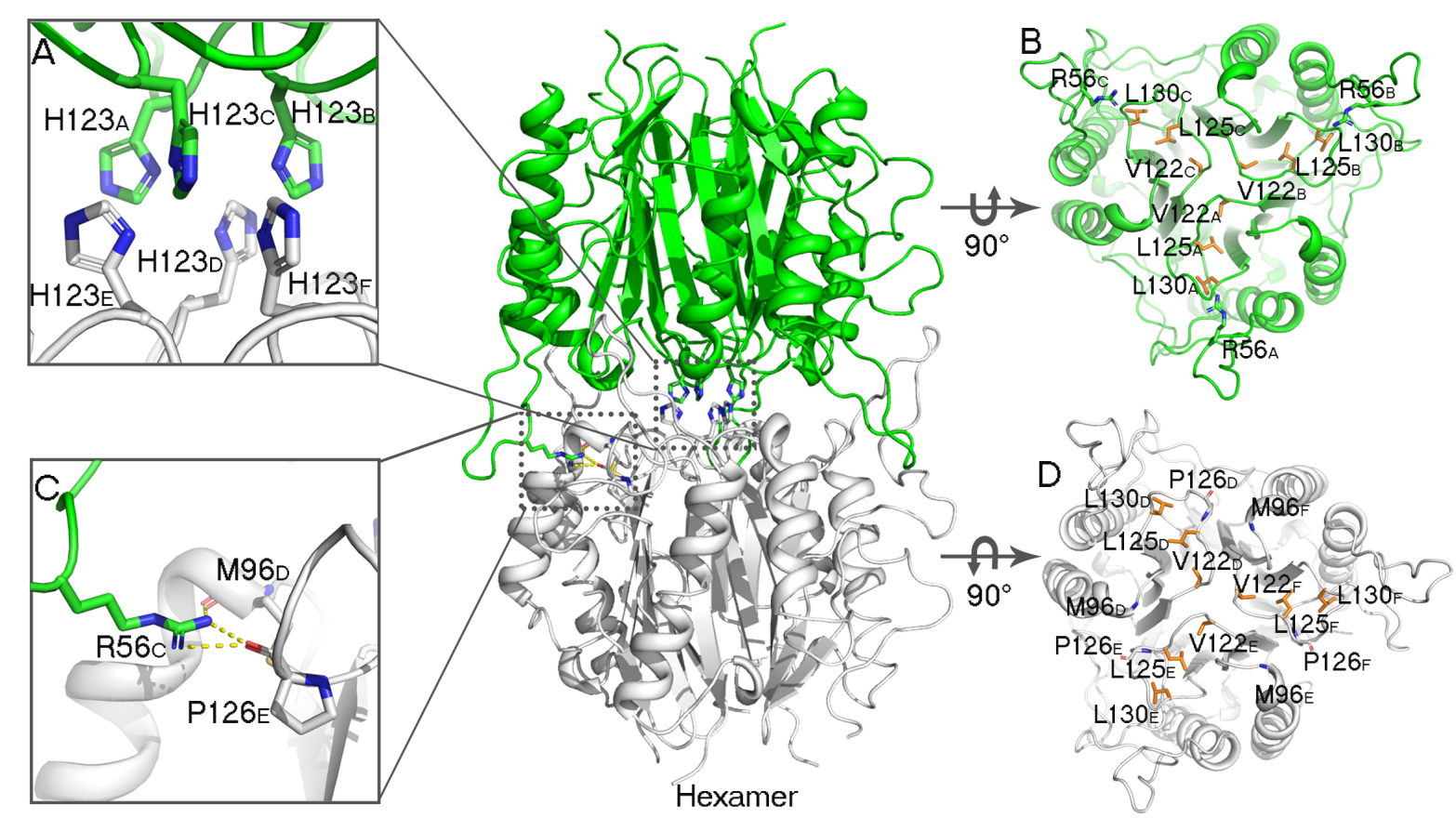

FIGURE 4 | Analyses of AmnE hexameric interface. (A) Details of six His ${ }^{123}$ located on the Lp3 are shown using sticks. (B) Hydrophobic loop Lp3 in trimeric structures of AmnE, the hydrophobic residues $\left(\mathrm{Nal}^{122}\right.$, Leu ${ }^{125}$, and $\left.\mathrm{Leu}^{130}\right)$ are shown in orange sticks. The three residues of Arg ${ }^{56}$ that participate in hydrogen-bond formations in the hexameric structure are shown in green sticks. (C) Details of hydrogen bonds connecting Arg ${ }^{56}$ with Met ${ }^{96}$ and Pro ${ }^{126}$ are shown using sticks. (D) Hydrophobic loop Lp3 in the trimeric structures of AmnE and the hydrophobic residues $\left(\mathrm{Val}^{122}{ }^{12} \mathrm{Leu}^{125}\right.$, and Leu ${ }^{130}$ ) are shown in orange sticks. The residues of $\mathrm{Met}^{96}$ and Pro ${ }^{126}$ that participate in hydrogen bond formations in the hexameric structure are shown in white sticks. The figure was produced with PyMOL (http://www.pymol.org).

RidA subfamily proteins has identified several residues in AmnE, including $\mathrm{Ser}^{35}, \mathrm{Gly}^{36}, \mathrm{Glu}^{88}, \mathrm{Gln}^{103}, \mathrm{Arg}^{117}$, and Lys ${ }^{135}$, which are highly conserved in clefts located at the subunit interfaces of the trimer (Supplementary Figure S8A). This indicates that these proteins play a similar role in cells. Lambrecht et al. revealed that RidA subfamily proteins exhibit enamine/imine deaminase activity and accelerate the release of ammonia from reactive enamine/imine intermediates (Lambrecht et al., 2010). Parsons et al. identified several compounds that interacted separately with the RidA subfamily protein HI0719 and protein hp14.5 in a pocket located in the clefts of the trimer separately (Parsons et al., 2003; Manjasetty et al., 2004). Therefore, the trimerization of the RidA subfamily proteins is necessary for achieving proper functionality in vivo. However, AmnE adopts homohexameric forms both in crystal structures and in solution states. A DALI search in the PDB library retrieved two similar hexameric structures (PDB code: $3 \mathrm{KJK}, 2 \mathrm{EWC}$ ) based on the structure of the AmnE hexamer. These two proteins were identified as Nmb1025 in Neisseria Meningitidis and SPy_2060 in Streptococcus Pyogenes M1 Gas. All three proteins adopted hexameric assembly, which comprised two identical trimers in a back-to-back configuration forming the hydrophobic base in the middle, and six hydrophobic loops in the center of the hexamer (Figures 6A,B,E,F). Meanwhile, 15-21 hydrogen bonds around the hydrophobic base connected the two trimers together and maintained a stable hexameric structure. The hexameric structures of the proteins Nmb1025, SPy_2060, and AmnE, were analyzed by PDBePISA (Supplementary Table S1). All three proteins formed a hydrophobic base in loop Lp3 that was composed of the highly conserved residues Val ${ }^{122}$, Leu ${ }^{125}$, Pro ${ }^{126}$, Pro $^{128}$, and Leu ${ }^{130}$ on the AmnE loop Lp3, as verified by sequence alignment in RidA subfamily (Supplementary Figure S8A). In addition to the hydrophobic base in the center of the hexamer, the Nmb1025 hexamer recruited residues $\mathrm{Arg}^{68}$, $\mathrm{Asp}^{69}, \mathrm{Glu}^{96}$, and $\mathrm{Arg}^{98}$ from each of the protomers, form hydrogen bonds (Figure 6C). The structures of the hypothetical protein Spy_2060 formed five hydrogen bonds based on the $\mathrm{Asp}^{72}, \mathrm{Gln}^{98}, \mathrm{Glu}^{100}$, and Gly ${ }^{106}$ from each of the protomers (Figure 6D). Therefore, the stable hexamer requires the hydrophobic base with 15-21 hydrogen bonds around it to connect the two trimers together. However, these residues forming the hydrogen bonds in three hexameric structures are not highly conserved in RidA subfamily. This is probably the reason why most of members in RidA subfamily adopt a trimeric form instead of hexameric conformation. We constructed a multigene expression system including amnA, amnB, and amnC genes from Pseudomonas sp. $A P-3$ and expressed in $E$. coli to produce 2-aminomuconate in order to test the enzyme activity of hexameric AmnE and trimeric variants due to lack of stable substrate and cells of Pseudomonas sp. AP-3. However, we unable to get the 2-aminomuconate due to its high instability. Therefore, the hexameric structure of AmnE presented is the first step to understanding the molecular 


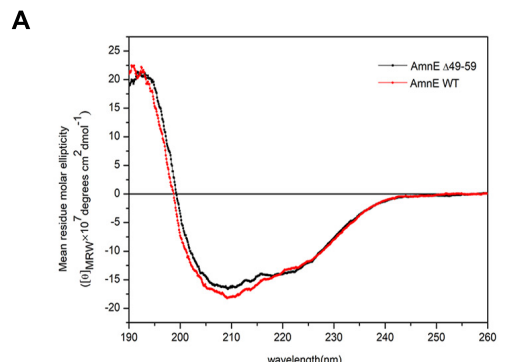

D
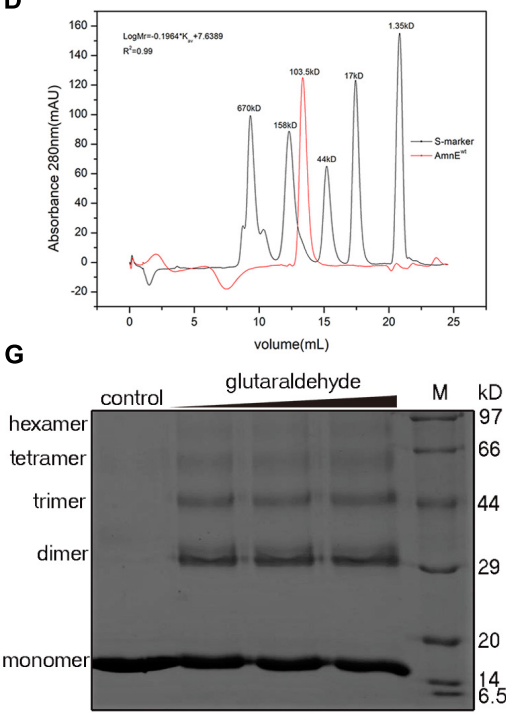

B

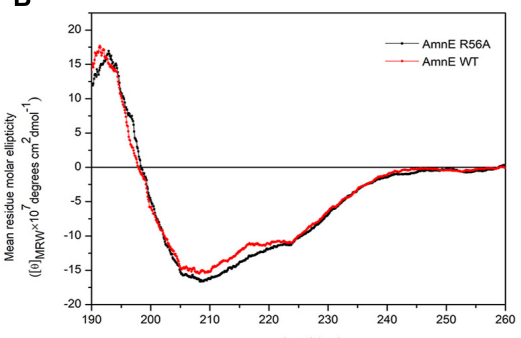

E

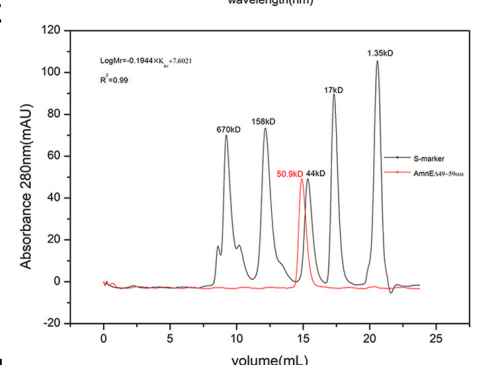

H

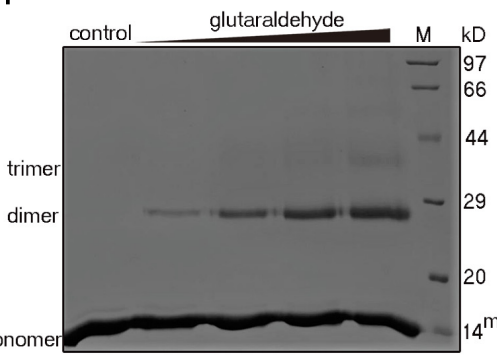

C

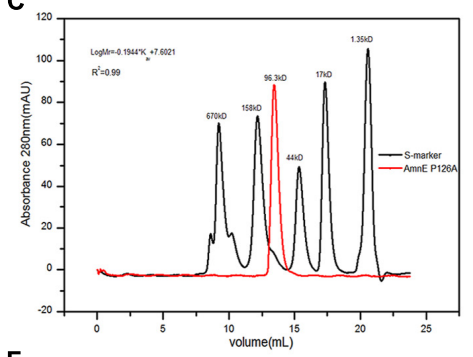

$\mathbf{F}$

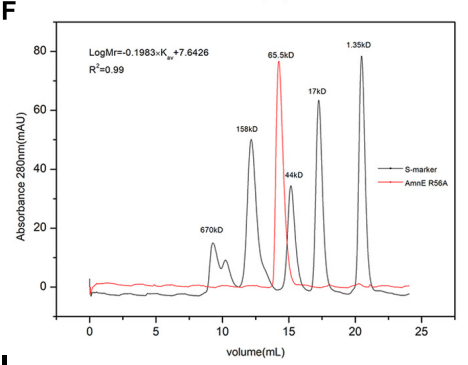

I

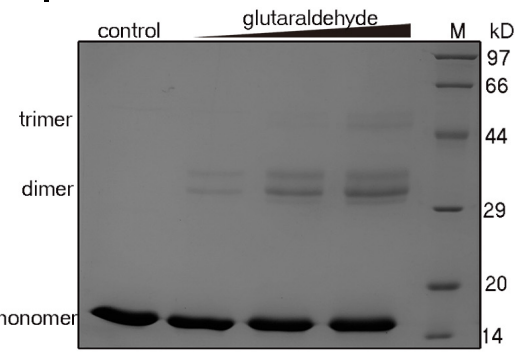

FIGURE 5 | Biochemical characterization of AmnE hexamerization. (A) Measurement of AmnE 4 49-59 (black line) and wild-type AmnE (red line) based on CD spectroscopy. (B) Measurement of AmnE R56A (black line) and wild-type AmnE (red line) based on CD spectroscopy. (C) Analytic size-exclusion chromatography of mutant AmnE P126A. The red solid curve refers to the AmnE P126A and the black solid curve refers to the molecular weight standards. (D) Analytic size-exclusion chromatography of wild-type AmnE. The red solid curve refers to the AmnE and the black solid curve refers to the molecular weight standards. (E) Analytic size-exclusion chromatography of mutant AmnE $\Delta 49-59$. The red solid curve refers to AmnE $\Delta 49-59$ and the black solid curve refers to the molecular weight standards. (F) Analytic size-exclusion chromatography of mutant AmnE R56A. The red solid curve refers to AmnE R56A and the black solid curve refers to the molecular weight standards. (G) Crosslinking of wild-type AmnE with increasing concentrations of glutaraldehyde are shown on SDS-PAGE gel, untreated wild-type AmnE loaded as control. In this and subsequent subfigures, $\mathrm{M}$ denotes a protein marker. $\mathbf{( H )}$ Cross-linking assay used to detect the polymerization forms of mutant AmnE $\Delta 49$ - 59. Crosslinking of AmnE $\Delta 49$ - 59 with increasing concentration of glutaraldehyde are shown on SDS-PAGE gel, untreated AmnE $\Delta 49$ - 59 loaded as control. (I) Cross-linking assay used to detect the polymerization forms of mutant AmnE R56A. Crosslinking of AmnE R56A with increasing concentration of glutaraldehyde are shown on SDS-PAGE gel, untreated AmnE R56A loaded as control.
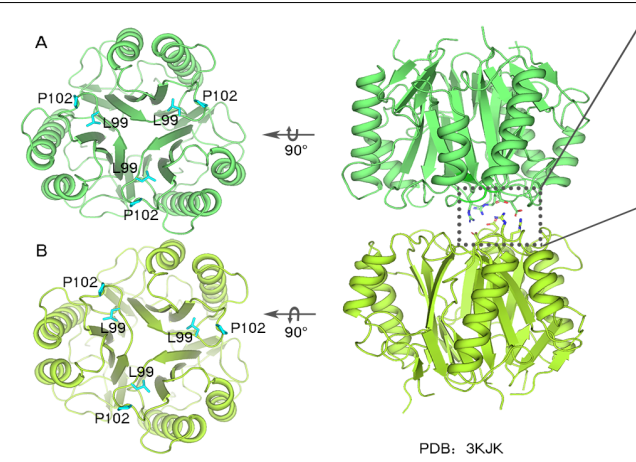

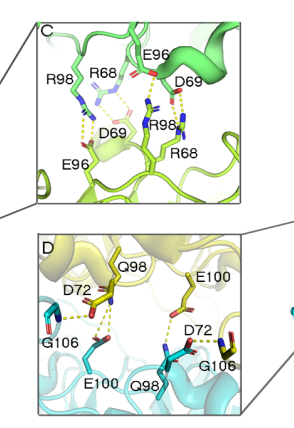

PDB: $3 K J K$

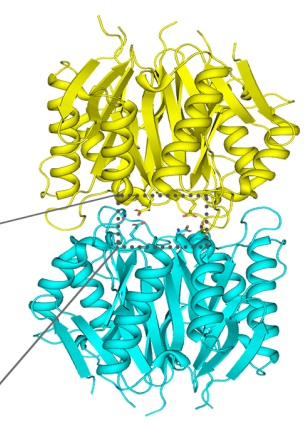

PDB: 2EWC

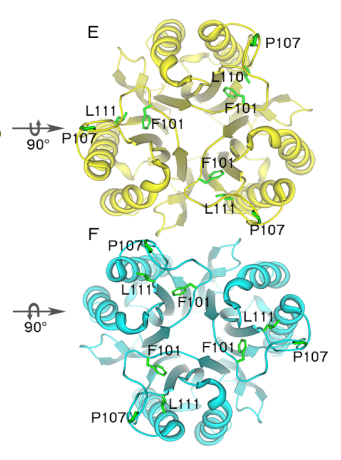

FIGURE 6 | Analyses of Nmb1025 (PDB: 3KJK) and hypothetical protein SPy_2060 (PDB: 2EWC) hexameric structure interfaces. (A,B) Hydrophobic loop Lp3 in the trimeric structures of $\mathrm{Nmb1025}$ in which the hydrophobic residues (Leu ${ }^{99}$ and Pro ${ }^{102}$ ) are shown using blue sticks. (C) Details of hydrogen bond formation of hexameric Nmb1025. The participating residues (Arg ${ }^{68}, \mathrm{Asp}^{69}$, Glu ${ }^{96}$, and Arg ${ }^{98}$ ) are highlighted with sticks. (D) Details of hydrogen bond formation of hexameric protein SPy_2060. Participating residues (Asp ${ }^{72}$, Gln ${ }^{98}$, Glu ${ }^{100}$, and Gly ${ }^{106}$ ) are highlighted as sticks. (E,F) Hydrophobic loop Lp3 in trimeric structures of protein SPy_2060, whereby the hydrophobic residues (Phe ${ }^{101}$, Pro ${ }^{107}$, and Leu ${ }^{111}$ ) are shown in green sticks. The figure was produced with PyMOL (http://www.pymol.org). 
mechanism of the different oligomerization types and their relation to their enzyme activity or substrate specificity in the modified meta-cleavage pathway.

\section{DATA AVAILABILITY}

Protein Data Bank Accession Codes: the atomic coordinates and structural factors of AmnE were submitted to the RCSB Protein Data Bank. The accession code is $6 \mathrm{IZH}$.

\section{AUTHOR CONTRIBUTIONS}

DS and YA-C conceived the experimental study, designed the experiments, analyzed the data, and wrote the manuscript. $\mathrm{HJ}$ and DL designed the fast-cloning protocol and assisted YA-C in the preparation of the expression vectors. YA-C and $\mathrm{TH}$ expressed and purified the wild type and mutants of AmnE. YA-C crystallized AmnE. YA-C and YI-C collected the X-ray diffraction data. DS and YI-C determined and analyzed the crystal structures. YA-C measured the multimerization of the AmnE and mutants by cross-linking and gel-filtration experiments. YR, $\mathrm{BY}, \mathrm{GB}$, and HD provided the material, helped to design the experiments, and analyzed data. DS reviewed and provided the approval for the submission/publication of the manuscript.

\section{REFERENCES}

Adams, P. D., Afonine, P. V., Bunkoczi, G., Chen, V. B., Davis, I. W., Echols, N., et al. (2010). PHENIX: a comprehensive Python-based system for macromolecular structure solution. Acta Crystallogr. D Biol. Crystallogr. 66(Pt 2), 213-221. doi: 10.1107/S0907444909052925

Deaconescu, A. M., Roll-Mecak, A., Bonanno, J. B., Gerchman, S. E., Kycia, H., Studier, F. W., et al. (2002). X-ray structure of Saccharomyces cerevisiae homologous mitochondrial matrix factor 1 (Hmf1). Proteins 48, 431-436. doi: 10.1002/prot.10151

Deriu, D., Briand, C., Mistiniene, E., Naktinis, V., and Grutter, M. G. (2003). Structure and oligomeric state of the mammalian tumour-associated antigen UK114. Acta Crystallogr. D Biol. Crystallogr. 59(Pt 9), 1676-1678. doi: 10.1107/ s0907444903014306

Emsley, P., and Cowtan, K. (2004). Coot: model-building tools for molecular graphics. Acta Crystallogr. D Biol. Crystallogr. 60(Pt 12 Pt 1), 2126-2132. doi: $10.1107 /$ S0907444904019158

He, Z., and Spain, J. C. (1998). A novel 2-aminomuconate deaminase in the nitrobenzene degradation pathway of Pseudomonas pseudoalcaligenes JS45. J. Bacteriol. 180, 2502-2506.

Hodge-Hanson, K. M., and Downs, D. M. (2017). Members of the Rid protein family have broad imine deaminase activity and can accelerate the Pseudomonas aeruginosa $\mathrm{D}$-arginine dehydrogenase (DauA) reaction in vitro. PLoS One 12:e0185544. doi: 10.1371/journal.pone.0185544

Kim, J. M., Yoshikawa, H., and Shirahige, K. (2001). A member of the YER057c/yjgf/Uk114 family links isoleucine biosynthesis and intact mitochondria maintenance in Saccharomyces cerevisiae. Genes Cells 6, 507-517. doi: 10.1046/j.1365-2443.2001.00443.x

Lambrecht, J. A., Browne, B. A., and Downs, D. M. (2010). Members of the YjgF/YER057c/UK114 family of proteins inhibit phosphoribosylamine synthesis in vitro. J. Biol. Chem. 285, 34401-34407. doi: 10.1074/jbc.M110. 160515

Lebedev, A. A., Vagin, A. A., and Murshudov, G. N. (2008). Model preparation in MOLREP and examples of model improvement using X-ray data.

\section{FUNDING}

This work was supported by grants from the National Key R\&D Programmes of China (2017YFA0505903), the National Natural Science Foundation of China (31370735 and 31670737), and the Sichuan Province Foundation (2014KJT021-2014SZ and 2015JQO029), and from the Science and Technology Department of Sichuan Province and Foundation (2016XT00-00033-GX-01) from the Chengdu HI-TECH Industrial Development Zone.

\section{ACKNOWLEDGMENTS}

We gratefully acknowledge the core facility staff members of West China Hospital. We thank the staffs from BL19U1 Beamline of National Facility for Protein Science in Shanghai (NFPS) at Shanghai Synchrotron Radiation Facility, for assistance during data collection.

\section{SUPPLEMENTARY MATERIAL}

The Supplementary Material for this article can be found online at: https://www.frontiersin.org/articles/10.3389/fmicb. 2019.02079/full\#supplementary-material

Acta Crystallogr. D Biol. Crystallogr. 64(Pt 1), 33-39. doi: 10.1107/ S0907444907049839

Li, C., Wen, A., Shen, B., Lu, J., Huang, Y., and Chang, Y. (2011). FastCloning: a highly simplified, purification-free, sequence- and ligation-independent PCR cloning method. BMC Biotechnol. 11:92. doi: 10.1186/1472-675011-92

Manjasetty, B. A., Delbruck, H., Pham, D. T., Mueller, U., Fieber-Erdmann, M., Scheich, C., et al. (2004). Crystal structure of Homo sapiens protein hp14.5. Proteins 54, 797-800. doi: 10.1002/prot.10619

Niehaus, T. D., Gerdes, S., Hodge-Hanson, K., Zhukov, A., Cooper, A. J., ElBadawi-Sidhu, M., et al. (2015). Genomic and experimental evidence for multiple metabolic functions in the RidA/YjgF/YER057c/UK114 (Rid) protein family. BMC Genomics 16:382. doi: 10.1186/s12864-0151584-3

Otwinowski, Z., and Minor, W. (1997). Processing of X-ray diffraction data collected in oscillation mode. Methods Enzymol. 276, 307-326. doi: 10.1016/ s0076-6879(97)76066-x

Parsons, L., Bonander, N., Eisenstein, E., Gilson, M., Kairys, V., and Orban, J. (2003). Solution structure and functional ligand screening of HI0719, a highly conserved protein from bacteria to humans in the YjgF/YER057c/UK114 family. Biochemistry 42, 80-89. doi: 10.1021/bi020541w

Schmiedeknecht, G., Kerkhoff, C., Orso, E., Stohr, J., Aslanidis, C., Nagy, G. M., et al. (1996). Isolation and characterization of a $14.5-\mathrm{kDa}$ trichloroaceticacid-soluble translational inhibitor protein from human monocytes that is upregulated upon cellular differentiation. Eur. J. Biochem. 242, 339-351. doi: 10.1111/j.1432-1033.1996.0339r.x

Sinha, S., Rappu, P., Lange, S. C., Mantsala, P., Zalkin, H., and Smith, J. L. (1999). Crystal structure of Bacillus subtilis YabJ, a purine regulatory protein and member of the highly conserved YjgF family. Proc. Natl. Acad. Sci. U.S.A. 96, 13074-13079. doi: 10.1073/pnas.96.23.13074

Takenaka, S., Murakami, S., Kim, Y. J., and Aoki, K. (2000). Complete nucleotide sequence and functional analysis of the genes for 2-aminophenol metabolism from Pseudomonas sp. AP-3. Arch. Microbiol. 174, 265-272. doi: 10.1007/ s002030000203 
Takenaka, S., Murakami, S., Shinke, R., and Aoki, K. (1998). Metabolism of 2-aminophenol by Pseudomonas sp. AP-3: modified meta-cleavage pathway. Arch. Microbiol. 170, 132-137. doi: 10.1007/s00203005 0624

Takenaka, S., Murakami, S., Shinke, R., Hatakeyama, K., Yukawa, H., and Aoki, K. (1997). Novel genes encoding 2-aminophenol 1,6-dioxygenase from Pseudomonas species AP-3 growing on 2-aminophenol and catalytic properties of the purified enzyme. J. Biol. Chem. 272, 14727-14732. doi: $10.1074 /$ jbc.272.23.14727

Volz, K. (1999). A test case for structure-based functional assignment: the $1.2 \mathrm{~A}$ crystal structure of the yjgF gene product from Escherichia coli. Protein Sci. 8, 2428-2437. doi: 10.1110/ps.8.11.2428

Winn, M. D., Ballard, C. C., Cowtan, K. D., Dodson, E. J., Emsley, P., Evans, P. R., et al. (2011). Overview of the CCP4 suite and current developments.
Acta Crystallogr. D Biol. Crystallogr. 67(Pt 4), 235-242. doi: 10.1107/ S0907444910045749

Conflict of Interest Statement: The authors declare that the research was conducted in the absence of any commercial or financial relationships that could be construed as a potential conflict of interest.

Copyright (C) 2019 Chen, Chen, Jiang, Lu, Hu, Bi, Ran, Yu, Dong and Su. This is an open-access article distributed under the terms of the Creative Commons Attribution License (CC BY). The use, distribution or reproduction in other forums is permitted, provided the original author(s) and the copyright owner(s) are credited and that the original publication in this journal is cited, in accordance with accepted academic practice. No use, distribution or reproduction is permitted which does not comply with these terms. 